\title{
Bioreactor for microalgal cultivation systems: strategy and development
}

\begin{abstract}
Microalgae are important natural resources that can provide food, medicine, energy and various bioproducts for nutraceutical, cosmeceutical and aquaculture industries. Their production rates are superior compared to those of terrestrial crops. However, microalgae biomass production on a large scale is still a challenging problem in terms of economic and ecological viability. Microalgal cultivation system should be designed to maximize production with the least cost. Energy efficient approaches of using light, dynamic mixing to maximize use of carbon dioxide $(\mathrm{CO} 2)$ and nutrients and selection of highly productive species are the main considerations in designing an efficient photobioreactor. In general, optimized culture conditions and biological responses are the two overarching attributes to be considered for photobioreactor design strategies. Thus, fundamental aspects of microalgae growth, such as availability of suitable light, $\mathrm{CO} 2$ and nutrients to each growing cell, suitable environmental parameters (including temperature and $\mathrm{pH}$ ) and efficient removal of oxygen which otherwise would negatively impact the algal growth, should be integrated into the photobioreactor design and function. Innovations should be strategized to fully exploit the wastewaters, flue-gas, waves or solar energy to drive large outdoor microalgae cultivation systems. Cultured species should be carefully selected to match the most suitable growth parameters in different reactor systems. Factors that would decrease production such as photoinhibition, self-shading and phosphate flocculation should be nullified using appropriate technical approaches such as flashing light innovation, selective light spectrum, light-CO2 synergy and mixing dynamics. Use of predictive mathematical modelling and adoption of new technologies in novel photobioreactor design will not only increase the photosynthetic and growth rates but will also enhance the quality of microalgae composition. Optimizing the use of natural resources and industrial wastes that would otherwise harm the environment should be given emphasis in strategizing the photobioreactor mass production. To date, more research and innovation are needed since scalability and economics of microalgae cultivation using photobioreactors remain the challenges to be overcome for large-scale microalgae production.
\end{abstract}

Keyword: Energy efficiency; Mathematical modelling; Microalgae; Nutrients; Photobioreactors; Solar energy 